\title{
INHALT
}

VORWORT

Vanessa Miriam Carlow

PROLOG

Marco Hemmerling, Boris Bähre

INFORMIERTE ARCHITEKTUR 10

BUILDING INFORMATION MODELLING

PRAXIS 1

MERCEDES-BENZ MUSEUM

30

Stuttgart

ORGANISATION

46

LIFECYCLE

BIM IM BESTAND

FRONTLOADING

BIM - BAM - BOOM

DIGITAL TWIN

RICHTLINIEN

EIR - BEP - MIDP

LOD - LOG - LOI

DIMENSIONEN

59

SIZE MATTERS?

\section{PRAXIS 2}

ASTOC ARCHITECTS AND PLANNERS

Köln

BARKOW LEIBINGER

Berlin

EIKE BECKER ARCHITEKTEN 
TECHNOLOGIE $\quad 92$

MODELLIERUNG 93

LEVELS OF BIM

LITTLE - BIG - CLOSED - OPEN 96

COMMON DATA ENVIRONMENT 98

DATENAUSTAUSCH 99

BIM-SOFTWARE 101

PRAXIS 3

DELUGAN MEISSL

ASSOCIATED ARCHITECTS 104

Wien

EM2N

116

Zürich

$\begin{array}{ll}\text { SOZIOLOGIE } & 128\end{array}$

EIN SOZIOTECHNISCHES SYSTEM 129

VERTRAUEN 130

NEUE ROLLEN 131

FEHLERKULTUR 134

BIM-SPRACHE UND -VOKABULAR 134

\section{LEHRE \& FORSCHUNG}

MIRCO BECKER 136

Leibniz Universität Hannover

JAKOB BEETZ 146

RWTH Aachen

ULRICH BLUM, HENRIETTE STROTMANN 154

FH Münster

EPILOG

Peter Russel

GLOSSAR 166

LITERATURVERZEICHNIS 172

ABBILDUNGSVERZEICHNIS $\quad 174$

AUTOREN $\quad 175$ 